\title{
Extensión en ciencias de la salud. Plan de acción social. Universidad de Carabobo, Venezuela
}

\author{
José Corado \\ Everilda Arteaga \\ Rosa Cristina Pérez \\ María Karina Arvelo \\ Ariel Reyes \\ Gilberto Bastidas
}

Facultad de Ciencias de la Salud. Universidad de Carabobo. Venezuela.

Email: bastidasprotozoo@hotmail.com

Resumen: En esta redacción se hace una breve descripción de la acción social o comunitaria de la medicina de atención primaria en salud y del porque y para que de nuevas estrategias de intervencion, en este caso, el Plan de Acción Social, de la Dirección de Extensión de la Facultad de Ciencias de la Salud, de la Universidad de Carabobo, en la labor de extensión y servicio comunitario que deben realizar todas y cada una de las universidades del país por estar consagrada en el estamento legal venezolano y por constituir la razón de ser de las instituciones de educación superior.

Palabras clave: medicina comunitaria, plan de acción social, promoción para la salud, atención primaria, extensión universitaria.

\section{Extension in health sciences. Social Action Plan. Carabobo University, Venezuela}

\begin{abstract}
In this paper we present a brief description of social or community action, of primary health care medicine, and the why and what for of new strategies of intervention, in this case, the Social Action Plan of the Extension Board of the Faculty of Health Sciences, University of Carabobo, in regards to the extension and community service each and every one of the universities in the country must undertake, as enshrined in the Venezuelan legal order and for constituting the raison d'être of superior education institutions.

Key words: community medicine, social action plan, health promotion, primary care, university extension.
\end{abstract}




\section{Extensão em ciências da saúde. Plano de Ação Social. Universidade de Carabobo, Venezuela}

Resumo: Neste artigo se faz uma breve descrição da ação social ou comunitaria do sistema de atenção primária de saúde e das razões das novas estratégias de intervenção, neste caso, o Plano de Acção Social, da Diretoria de Extensão da Faculdade de Ciências da Saúde, Universidade de Carabobo, na sua tarefa de extensão e serviço comunitário a ser realizada por cada uma das universidades do país, pois se encontra consagrada no ordemanento legal da Venezuela e, por constituir a essencia das instituições do ensino superior.

Palavras-chave: medicina comunitária, plano de ação social, promoção da saúde, atenção primária de saúde, extensão universitária.

$* * *$

\section{Introducción}

En el país se producen constantes cambios sociales, culturales, económicos y políticos, que obligan a la reinvención en la forma de abordar las crisis que estos generan, particularmente en la salud de las comunidades, porque su contraparte la enfermedad impide el desarrollo humano adecuado y por ende el social. Por tanto, se requiere que los entes encargados de administrar la salud, la atención de la misma, sea del sector oficial, privado o universitario se adentren en las comunidades, que salgan del intramuro, que se empapen de las necesidades sentidas como tal por las personas que viven allí, donde el papel de las universidades es crucial, dada su intrínseca función de docencia, extensión e investigación en la formación de profesionales en ciencias de la salud, máxime porque la diversidad de necesidades requiere del implemento de variadas posibilidades de medidas para la satisfacción de las mismas, claro está en consonancia directa con los diferentes escenarios de realización personal, inserción comunitaria e interrelación con el entorno, ya que las personas y por ende las comunidades son diferentes entre sí, diferencias signada por las características innatas y adquiridas de cada quién y por condicionantes que pueden comportase según el caso como favorecedores o desalentadores del proceso de satisfacción de necesidades, en este caso, la salud (Arouca, 1975; McWhinney, 1981; Owens, Dominici y Saldaña, 1998; Silva, 2009).

En un mundo grandemente poblado donde la incorporación irracional de la alta tecnología en la salud, sin criterio y por supuesto sin lógica epidemiológica, genera por un lado, aumento de la inequidad y baja calidad de vida, debido al importante incremento de los costos, y por tanto, en las restricciones para el acceso a parte de la sociedad, la pobre, la de menor recursos; y por otro lado, crea en la sociedad la tendencia a priorizar la especialización "la medicina por aparatos y sistemas" se entiende que esta sea necesaria pero no debe ser el centro de la atención en salud, debe serlo la promoción. No se ve la salud como un proceso dinámico de gran variabilidad en que intervienen factores biológicos, psicológicos, ambientales y 
sociales, y menos aún se ve la salud ligada al concepto de bienestar, como el conjunto de acciones dirigidas a favorecer el desarrollo humano y el bienestar individual y comunitario, a través, de la promoción de hábitos saludables, control de factores de riesgo, preservación del medio ambiente y desarrollo de medidas de recuperación y rehabilitación frente a la agresión (Arouca, 1975; González, 2004; McWhinney, 1981; Owens, Dominici y Saldaña, 1998; Silva, 2009).

Entonces la salud debe centrarse hacia el enfoque de riesgo en epidemiologia, como predicción cuantificable de causalidad sobre el conocimiento de la relación entre exposición y efecto, pero signado por incertidumbre, con base en la probabilidad pero en dependencia directa de las contingencias, porque no siempre se conocen los mecanismos del proceso, no obstante se observan sistemáticamente series de hechos que implican estabilidad de patrones de ocurrencia de eventos de efectos positivos o negativos, con el propósito de describir el futuro deseable, a través de la consideración de que puede ganarse o perderse con la decisión tomada, no solo en salud, sino también en la sociedad, en la búsqueda del altamente ponderado “estilo de vida saludable”, el de los grupos como el todo homogéneo, pero sin dejar de lado al hombre como ser único y distinto (De Almeida, Castiel y Ayres, 2009).

Es igualmente necesario pasar del tradicional enfoque reduccionista y fragmentado de la salud hacia una nueva propuesta de abordaje centrada en una vertiente de análisis más interpretativa, que posibilite la comprensión del significado, es decir, la contextualización de los hechos. (De Almeida, 2000). Es así que paulatinamente se ha convenido que el reduccionismo, que busca subyugar el todo a sus partes más pequeñas, se encuentra verdaderamente fuera de lugar (Stewart, 2001). Por ello se apuesta por el enfoque holístico como alternativa al reduccionismo, pero el que ve el aspecto global sin detrimento del particular, como un proceso de integración, y desagregación con conservación de las relaciones entre los componentes, entendido como pensamiento complejo, es decir, que distingue sin desunir (Smuts, 1926; Weil, 1990; Morin, 2003), en este mismo sentido Weinstein (1991), señala que "Salud es lo que somos: es el grado de actualización de nuestras capacidades, de integrarnos en las distintas dimensiones que nos conciernen”.

En este contexto, la formación del recurso humano del sector adquiere relevancia como eje transformador de la práctica profesional actual que, desde hace tiempo, es cuestionada por no responder, aunque lo intenta, con la eficiencia y eficacia indispensable a las reales necesidades de los individuos insertos en su vida familiar y comunitaria. En este sentido, la reorientación de la formación del personal de salud debe basarse en el enfoque integral del proceso salud-enfermedad-atención y en sus determinantes; en el adecuado empleo de las herramientas tecnológicas; y en el contexto socio-cultural en que se enmarca y su organización en la práctica, es decir en la real y verdadera participación comunitaria (Arouca, 1975; McWhinney, 1981; Owens, Dominici y Saldaña, 1998; Silva, 2009). 
De esto se desprende la urgencia, ya hacia lo interno, en la universidad, por demás extensionista, de formar el talento humano para el sector salud con las comunidades, en la gestión del proceso de salud o en el autocuidado de la misma. Se precisa entonces la articulación y vinculación del modelo educativo a las necesidades y requerimientos de la sociedad, con base en la interdisciplinariedad, en la difusión y socialización de los conocimientos. Entonces para ser fieles a los tres pilares que soportan a la Universidad de Carabobo, el hombre, el conocimiento y la sociedad, nace de la Facultad de Ciencias de la salud el Plan de Acción Social (PAS-FCS), como un intento que persigue articular la formación del talento humano para intentar dar repuestas a las necesidades en salud de las comunidades, con énfasis en el posicionamiento local del saber en salud, en la realidad social, en la suma de esfuerzos, interdisciplinariedad y enfoque humanístico.

\section{La acción social o comunitaria}

Se define a la acción social como cualquier tipo racional de proceder humano, que se orienta por el maniobrar de otros y es dirigido por el carácter subjetivo de un individuo o de grupos de ellos, para alcanzar un fin o un ideal. En la acción social se muestra al individuo como el actor quien efectúa el comportamiento orientado al logro, siempre bajo la influencia de objetos físicos (Los medios), sociales (Otros autores) y culturales (Principios normativos) e inmersos en tres sistemas; el de la personalidad, el actor con sus impulsos, actitudes y destrezas (interiorización del comportamiento); el social, centrado en el rol que nace de las expectativas, pero que implica relaciones normativas (institucionalización del comportamiento); finalmente el sistema cultural, a partir del cual se configura el comportamiento a desarrollarse dentro de los anteriores sistemas, es así, que en la sociedad moderna se muestra a la acción social como una realidad externa al individuo, donde la forma de cohesión, es la solidaridad orgánica, es decir, la interdependencia entre las distintas funciones de la especialización y singularidad de cada individuo en grupo (Parsons, 1951).

Por esto, y según Ferrara, Acebal y Paganini (1972), la acción social o comunitaria, ha sido introducida en el pensar y accionar profesional, a tal punto, que es a través de ella, que se ha jerarquizado la función social de las profesiones, aspecto que parece haber sido dejado de lado entre las profesiones universitarias relacionadas con la atención de la salud, pues es habitual que no se aborde, para el tratamiento de los problemas sanitarios, la comunidad estructuralmente, es decir, con límites geográficos, con cierta autonomía administrativa, con habitantes comprometidos en actividades interrelacionadas, movidos por intereses, aspiraciones y necesidades comunes, con desempeño de roles sociales en acuerdo con las expectativas de comportamientos establecidas por el grupo, mismas, que a su vez, están bajo la dirección de procesos de socialización y control (Lindman, 1951). La acción social representa una metamorfosis interminable, en que se crean necesidades, se satisfacen, pero inmediatamente se crean otras, puede hablarse, entonces, de intercambio creativo entre el hombre y su medio am- 
biente, un medio provisto de personalidad que generalmente determina el tipo de individuo y porque no de sociedad (Marx y Engels, 1959).

Pero ya es tradicional en el campo socio-sanitario el dejar de lado a la comunidad en lo que respecta a su salud, es decir, se les despoja o desvincula de la participación y titularidad de este derecho social, en consecuencia a menor relación social y ámbito de participación, menor será el capital social para enfrentar los problemas (Castel, 1995; Gacitúa y Davis 2000). Entonces es desde dentro de las comunidades que se puede y debe desarrollar la atención en salud, tamaño papel que debe cumplir la universidad extensionista, en la formación de los profesionales de la salud, porque las comunidades, específicamente el accionar de ellas, como conjunto de recursos actuales o potenciales en una red de relaciones institucionalizadas, por el mutuo conocimiento y reconocimiento, facilitaría el abordaje exitoso del proceso salud-enfermedad-atención, con base en los beneficios que representa la participación en grupos dada la deliberada sociabilidad para el propósito, mediante la inversión en estrategias que generarían beneficios socio-sanitarios.

\section{La medicina comunitaria es la atención primaria en salud}

Desde la conferencia de Alma-Ata de la Organización Mundial de la Salud en 1978, se define a la medicina comunitaria, como Atención Primaria de Salud (APS), es decir, el conjunto de estrategias, métodos y actividades orientadas a mejorar el estado de salud de una población definida, aquella que cae dentro del ámbito de trabajo de los equipos de APS y que puede ubicarse en espacios territoriales distintos, siempre y cuando se consideren a estos como un todo, es decir, una comunidad (Gofin y Foz, 1998; Jiménez-De Gracia, Ruiz-Moral, Gavilán-Moral, Hueso-Montoro y CanoCaballero, 2012).

La medicina comunitaria tiene hoy día gran auge debido al favorable impacto que sobre la salud produce y por el desarrollo de metodología propia, porque su elemento conceptual es la práctica de la atención primaria con orientación a la salud de toda la comunidad y con la participación de la misma en todas las fases del proceso; con base metodológica, en la equidad, en la práctica basada en la evidencia científica, en el trabajo por programas y por supuesto en el uso local de la epidemiología para identificar las necesidades de salud y en la constante evaluación de las intervenciones, como mecanismo, inequívoco de retroalimentación, ilustrado como el mecanismo que asegura la bondad del trabajo y la posibilidad de generar estimulo al logro de los objetivos (Abramson, 1984; 1988; Whright, 1993).

Específicamente, y como metodología propia, la medicina comunitaria inicia en una determinada región con el análisis de la situación de salud y la priorización de las urgencias más relevantes para la comunidad en referencia directa a las necesidades en salud sentidas como importantes para ese determinado grupo social, lo que permite al equipo de salud afron- 
tar, especialmente, aquellas cuyo condiciones de planificación garanticen la posibilidad de resolución de la necesidad. De la rigurosidad metodológica se desprende la imposibilidad de sustraerse a la práctica de la medicina comunitaria que tienen los profesionales, la administración de servicios de salud e incluso la población (como responsables de su propia salud), como consecuencia de las grandes potencialidades que presentan en la calidad de vida de los grupos sociales (Ruiz-Moral, Pérula, Muñoz, Jiménez, González, y Alba, 2011).

El enfoque de la medicina comunitaria hacia la salud basada en las necesidades sentidas de la población permitirá el ajuste de los programas de salud oficiales, los impuestos de arriba hacia abajo por la planificación central en salud, rígidos, que no aceptan cambios porque simplemente no fueron diseñados para detectar las variaciones, si se quiere normales en la historia natural de una determinada enfermedad (Andrade, 2007; Cashman, Anderson, Weisbuch, Schwarz y Fulmer, 1999; Magill, 1999; Nickens,1999; ). Vale concluir en virtud de lo hasta ahora señalado, la necesidad que las universidades, con base en su carácter extensionista, gradúen profesionales de salud en atención primaria con una visión holística del proceso salud-enfermedad-atención, en que la atención se base en el paciente como individuo y no solo en la enfermedad e igualmente en la familia o el entorno más inmediato del paciente como condicionante del estado de salud; que valore e impulse la participación comunitaria en la resolución de problemas sanitarios; y que resuelva, o al menos intente hacerlo, los problemas en salud reales de la comunidad, es decir, los verdaderamente sentidos como necesidades, y no los considerados como tal desde los entes oficiales y centrales de salud venezolano.

De allí, que debe romperse, ya se está en ello, con el enfoque tradicional de la atención de salud venezolana, la usualmente realizada en que el cuidado y responsabilidad de la atención a la comunidad recaía sobre servicios asistenciales (netamente curativos) mínimos e integrados solo por médicos y enfermeras (Portillo, 1994). Por tanto, es absolutamente pertinente que los profesionales de la salud entre ellos los formadores del equipo dispensador de salud, las autoridades sanitarias y la población, particularmente, esta última, le agreguen valor y les concedan el espacio necesario para su desarrollo en cualquier medio, el rural o el urbano, siempre en bien de la comunidad, en la consecución de calidad de vida para todos y cada uno de los miembros de los grupos sociales como fin último de la medicina comunitaria (Mullan, 1982).

Es por eso, que en el actual pluralismo de salud debe romperse, ya se está en ello, con el enfoque tradicional y predominante de la atención de salud caracterizada por la continua expansión de la biomedicina basada en la investigación, en la producción farmacológica y en la indiscriminada medicalización de enfermedades y comportamientos, en total desmedro de otras formas de atención, especialmente la atención primaria en salud, la llamada medicina comunitaria, cuya base principal es la promoción de la salud y la protección específica, con carácter holístico y gran participación 
comunitaria, pues debe recordase, aunque en alusión a los distintos tipo de atención medica, pero que resalta el espíritu de diversidad, como afirmara Menéndez (2003), que en nuestras sociedades la mayoría de las poblaciones utilizan varias forma de atención no solo para diferentes enfermedades sino para una misma enfermedad. Por lo tanto, debe al menos reconocerse su existencia, sino puede dársele cabida, a la diversidad de la atención medica, dado que toda una serie de atenciones medicas de afecciones es negada, ignorada o marginada, a pesar de ser utilizadas por diferentes sectores de la comunidad.

\section{Los profesionales en salud. Integración docencia, extensión y comunidad}

Los paradigmas que direccionan la formación en salud han evolucionado, se han rediseñado hacia el análisis multicausal de los problemas, hacia la integración y el trabajo en equipo, entre distintas profesiones, es decir, hacia la perspectiva interdisciplinaria, con estrecha relación entre sistemas educativos y de salud cuyo centro es la persona, pero sin duda la agrupada en comunidad, como productores y conductores de necesidades y demandas ; también, los paradigmas, están orientados hacia la formación por competencias y a la validación del componente socio-cultural y hacia la búsqueda de respuestas razonadas que vayan de lo local a lo global en intima comunión con el desarrollo tecnológico. Pues, desde mediados del siglo pasado se ha transitado desde el enfoque esencialmente biológico del proceso salud-enfermedad-atención a las conceptualizaciones actuales que vinculan al proceso con variables o factores sociales, culturales, económicos y ambientales (Frenk, Chen, Bhutta, Cohen, Crisp y Evans, 2010; Bosi, 2012).

En este sentido, es de señalar que, las Escuelas de Medicina desarrollan una reforma en sus planes de estudios influenciadas, en primer lugar por el llamado informe Flexner (2010), donde, las ciencias modernas se organiza en el currículo sobre la base de dos ejes prioritarios: un ciclo de asignaturas básicas biomédicas, en las universidades, con fuerte contenido experimental y un ciclo clínico, en los hospitales, que incluye el internado rotatorio por las cuatro especialidades básicas (Cirugía, Gineco-Obstetrica, Medicina Interna y Pediatría). En segundo lugar, las reformas se ven influenciadas por el reporte Welch-Rose (1915), que ofrece dos nuevas visiones sobre la formación del profesional de salud, la de Rose, que plantea la creación de un sistema nacional de salud pública, con una escuela nacional y central, que actúa como el foco de una red estadal de escuelas; la visión de Welch, fue la de crear institutos de higiene con énfasis en la investigación científica y conectados a escuelas de medicina de las universidades.

De forma paralela a estos enfoques, de conocimiento e información, surge en las universidades por presión social la extensión universitaria, la sociedad como un todo exige a las casas de estudios superiores que no 
solo se compongan de estudiantes que aprenden y de profesores que enseñan, sino que entren en las comunidades, que se sintonicen con los pueblos donde están insertas, que sus profesionales tengan destrezas y competencias producto del diario vivir con sus congéneres, hombres y mujeres que viven en sociedad, que se traten como pares. Se necesita una universidad, y específicamente una Facultad de Ciencias de la Salud, en perfecto engranaje entre lo que la sociedad espera de ella y lo que realmente esta realiza, con base a la transdiciplinariedad en el análisis y la solución de problemas socio-sanitarios, y siempre reforzando sus funciones de servicio a la comunidad, es así que la extensión universitaria como promotora multidireccional de la interacción social se convierte en una poderosa herramienta para enfrentar con éxito las dificultades sociales en salud que se presenten en la sociedad actual, al poder influir sobre ella y por ende poder propiciar cambios (González, 2004).

El camino sin duda, es la formación hacia la resolución de problemas de una población definida, el desarrollo de competencias profesionales que demande la sociedad y la creación de sistemas sanitarios basados en la atención primaria en salud, sustentada esta última en la: atención accesible, detallada, coordinada, continua y responsable; al cual se debe añadir el componente comunitario y la aplicación de métodos epidemiológicos al cuidado clínico de pacientes individuales, para ello es necesario incorporar la medicina comunitaria y la atención primaria a las enseñanzas teóricas y prácticas de los currículos; apostar por la construcción colectiva y experiencial del aprendizaje, movilizar voluntades y garantizar recursos; y formar profesores y lideres docentes en medicina comunitaria. Todo esto con el fin de promover los cuidados de salud que el paciente necesita, cuando y como los requiera, con base en evidencia y siempre teniendo en cuenta que están condicionados por aspectos religiosos, étnicos, económicas y políticas prevalentes en las comunidades (Donabedian, 1990; World Health Organization, 2000; Menéndez, 2005; Organización Panamericana de la Salud, 2008).

$\mathrm{Al}$ respecto, y en mutuo acuerdo con Menéndez, es prudente considerar que el sector salud, y en particular los entes encargados de formar profesionales en medicina, especialmente las universidades, deben conocer y por supuesto implementar, o al menos adaptarse lo más fielmente posible, al tipo de atención real en salud que los grupos sociales aceptan, por supuesto sin que esto signifique el abandono de la enseñanza técnicacientífica, pero si la partida para establecer la existencia de afecciones y las formas de atención que las comunidades reconocen y utilizan, con base en los perfiles epidemiológicos y las estrategias de atención que desarrollan el saber biomédico, los curanderos tradicionales, los curanderos alternativos y los grupos sociales, de esto se desprende la necesidad de formar con y para las comunidades a los estudiantes de ciencias de la salud de las universidades, lo cual posibilitara conocer tanto las convergencias, como las divergencias de los tipos de atención utilizadas y del perfil epidemiológico dominante (Menéndez, 1981; Menéndez, 1984). 


\section{Plan de Acción Social de la Facultad de Ciencias de la Salud (PAS-FCS). Marco epistemológico}

El desafío actual está en articular las ciencias de la salud y la estructura social para orientar la formación del personal de salud, hacia la Medicina Comunitaria, claro está como la describiera, Sidney Kark, es decir, la atención primaria en salud orientada a la comunidad, con especial énfasis en aspectos sociales, culturales y del comportamiento del paciente atendido, con base en el enfoque holístico del individuo, familia y comunidad (Geiger, 1993; Silver 1984), surge entonces en el seno de la Facultad de Ciencias de la Salud de la Universidad de Carabobo, el Plan de Acción Social, con la pretensión de impulsar, aún más, la atención primaria integral, la holística, la orientada a la comunidad, como modelo que realmente de respuesta a las necesidades socio-sanitarias de las comunidades en el ámbito de la extensión universitaria, este plan persigue seguir el gigantesco ejemplo, al menos sembrar una semilla, del insigne Médico José Bengoa originario del país Vasco, quien se dedicó a entender y combatir la compleja interacción hambre-enfermedad cuyo punto de partida fueron los niños desnutridos del municipio Sanare, estado Lara, a él se debe la creación de los importantes Centros de Recuperación Nutricional de Venezuela, actualmente extendidos por todo el mundo (Alemay, Bernabeu-Mestre, Galiana, López y Trescastro-López, 2009).

El enfoque integral del proceso salud-enfermedad al cual se hace mención, implica: unidad de objetivos para realizar acciones en salud como función biológica y social, e interdisciplinariedad al analizar los fenómenos normales y patológicos del individuo y porque no de la comunidad con el aporte simultaneo y sistematizado de los principios y técnicas de distintas disciplinas, ciencias básicas, clínicas y sociales, para explicar el origen y lograr en primera instancia la prevención o al menos la curación. Se pretende, también y a groso modo, que el estudiante visualice la complejidad y especificidad de la práctica en salud orientada a la comunidad, que reflexione sobre los factores que condicionan esta tarea en cada contexto comunitario y sobre los efectos e impacto de los mismos.

Atención primaria que debe prestarse desde dentro de la comunidad, es decir, en el espacio del futuro trabajo profesional; en perfecto engranaje con la integración docencia-extensión-investigación universitaria; en la aplicación de la epidemiologia al proceso salud-enfermedad-atención; y en pulcra interacción entre ciencias biológicas y sociales. Para culminar con esta sección en la figura 1 se muestra la misión, objetivo y visión del PASFCS, enmarcados en dos actividades básicas: la primera, la promoción de la salud: centrada en la educación para la salud, específicamente, en temas relacionados con el cuidado y la preservación de la misma. Este primer conjunto de actividades, que encajan en el periodo pre patogénico de la historia natural de la enfermedad, tiene su asiento en las comunidades, en sus escuelas, iglesias, lugares de esparcimiento y demás sitios acondicionados para tal fin; y la segunda actividad ha referencia a los planes de 
intervención, a la acción, para recuperar la salud perdida, cuando fuere el caso, con base en el diagnóstico precoz y el tratamiento oportuno: mediante visitas de los actores universitarios (estudiantes de pre y postgrado, docentes y personal administrativo) a las comunidades y/o el traslado de las mismas, según el caso, a los centros de extensión de la Facultad de Ciencias de la Salud de la Universidad de Carabobo

Figura 1 Misión, objetivo y visión del Plan de Acción FCS-UC.

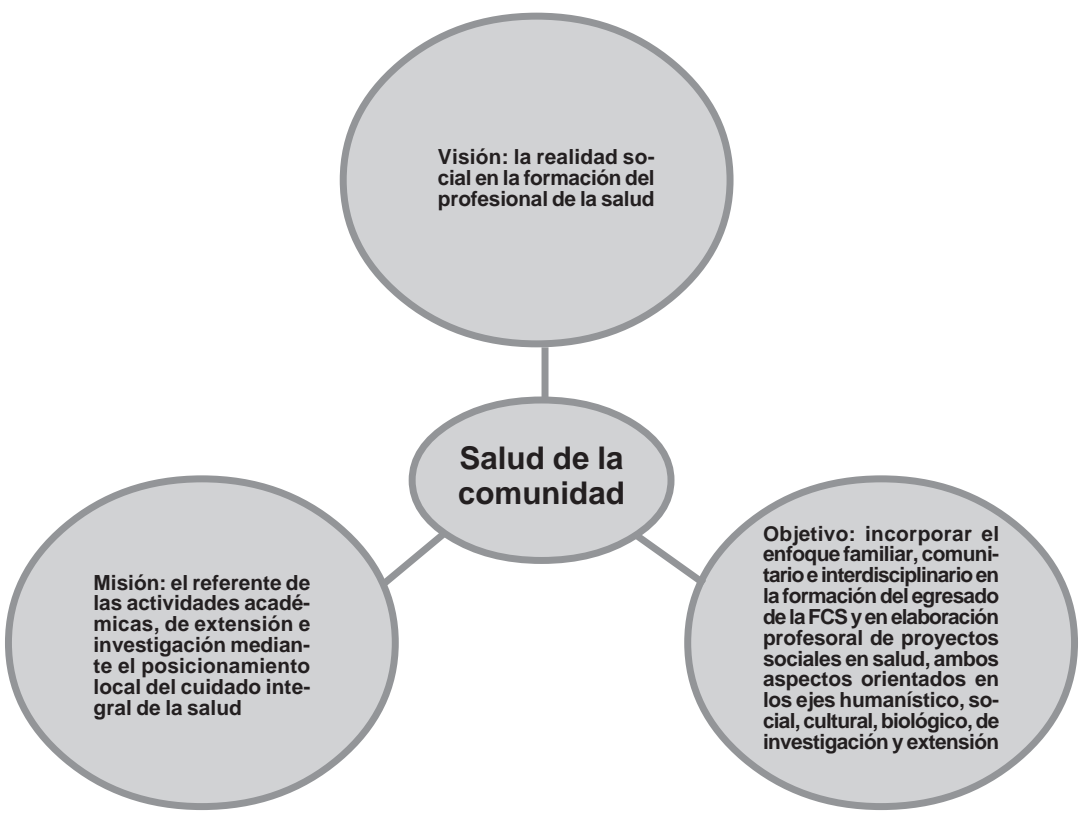

Fuente: Elaborado por los investigadores.

\section{La acción en el plan. 2010-2012}

Este plan de salud "PAS-FCS" que hace referencia a actividades académicas, de extensión e investigación mediante el posicionamiento local del cuidado de la salud, a través del trabajo con la comunidad comenzó para los estudiantes de la Facultad de Ciencias de la Salud-UC el 3 de mayo de 2010 en que se abordaron todos los hogares de las comunidades Brisas de Carabobo (730) y Simón Bolívar (699), ubicadas al Norte del estado Carabobo, igualmente se hizo con la Comunidad la Democracia II (362) al sur del estado. Entre las comunidades Brisas de Carabobo y Simón Bolívar se evaluaron integralmente, en visitas diarias casa por casa, 2.745 indivi- 
duos de ambos géneros, 1.118 en la primera y 1627 en la segunda. Ahora bien, en la comunidad La Democracia sector II se evaluaron 975 mujeres y hombres.

Asimismo, se recabó de los padres o jefes de familia información sobre: edad, género, parentesco, nivel educativo, ocupación, nivel socioeconómico, antecedentes patológicos familiares, personales y hábitos psicobiológicos (consumo de café, alcohol, tabaco o sustancias psicotrópicas), sobre su vivienda (características del techo, piso, paredes, número de habitaciones entre otros), servicios públicos (como: deposición de excretas, electricidad, fuentes de energía para cocinar, internet, agua para consumo humano y recolección de basura), sobre animales domésticos o que acarren riesgos para la salud, salud sexual y reproductiva, sobre esquema de inmunización, seguridad alimentaria, también se pidió información sobre diabéticos, hipertensos y patologías de la esfera genital de mujeres y hombres.

Luego de determinados los principales problemas de salud de las mencionadas comunidades y para dar respuestas a los mismos, es decir, para accionar en salud, se realizaron estudios coproparasitológicos y jornadas de desparasitación; y en el abordaje de las enfermedades crónicas no transmisibles y trastornos nutricionales, otros de los problemas de salud sentidos por las comunidades estudiadas se realizaron exámenes hematológicos; y en el caso de dislipidemia e hiperglucemia se efectuaron jornadas para la instauración del tratamiento farmacológico especializado totalmente gratuito y se inicia el seguimiento y control de las personas afectadas por estas patologías. Se comienza y con ahínco los trámites para la estructuración de laboratorios de Cardiorespiratorio y de citología, para ayudar, por un lado a detectar alteraciones de la esfera cardiorespiratoria, y por otro hacer detección precoz de cáncer cérvico-uterino, otras de las necesidades sentidas como problema de salud en las comunidades abordadas.

El PAS-FCS fundamentado en el primer nivel de atención en donde primordialmente se llevan a cabo todas las actividades de prevención y de promoción de la salud; en las grandes dificultades observadas para el acceso a los servicios de salud; en el bajo ingreso económico de las familias de comunidades; y el reducido nivel de escolaridad, ve como meta, pues el objetivo es ofrecer calidad de vida, mantener y profundizar la extensión de sus servicios de salud a toda la población, primero en el área de influencia de la universidad y luego a las poblaciones del resto del país que ameriten la intervención. En este sentido, se amplió para el año 2011 a 23 el número de comunidades atendidas y ya con la participación de estudiantes del segundo y sexto año de medicina, bioanálisis, citotecnología y cardiorespiratorio. De la misma manera los estudiantes de la asignatura Salud Comunitaria del segundo año de medicina establecieron salas situacionales con el fin de analizar la problemática socio-sanitaria que afecta al grupo social, generar lluvias de ideas y fomentar la participación comunitaria. 
El ámbito de acción de la Universidad de Carabobo, específicamente de la FCS, se extiende en el año 2011 en el marco del PAS hasta Araure en el estado Portuguesa, por petición de autoridades gubernamentales y eclesiásticas, quienes manifestaron la necesidad que tienen los pobladores de esta región de evaluaciones clínicas integrales. Ya para el año 2012 se suman al plan de acción social los estudiantes del sexto semestre de la carrera técnica Registros y Estadísticas de Salud en el vaciamiento de las historias familiares y procesamientos de datos socio-sanitarios de las comunidades y también los estudiantes de la carrera técnica en Imagenología en la realización e interpretación de estas pruebas diagnósticas. Para este año ya se atienden 34 comunidades de 8 Municipios en dos estados Carabobo y Cojedes. En estas líneas se intenta resumir las actividades que en tres años se realizaron con el PAS-FCS, en la búsqueda, del ideal, es decir, más y más calidad de vida para las comunidades, el fin último que persiguen las universidades del país con el compromiso de seguir atendiendo socio-sanitariamente a las comunidades.

El propósito de la propuesta en este papel redactada es plantear la instauración en el currículo de las carreras de ciencias de la salud de la Universidad de Carabobo, para servir de instrumento o al menos de refuerzo al servicio comunitario obligatorio de los estudiantes de educación superior, la acción social en salud, ya no como un plan de extensión universitaria, sino quizás como parte formal, debido fundamentalmente a que el biologismo, individualismo, la a sociabilidad, el mercantilismo y la eficacia pragmática definitorios de la practica medica del siglo XIX son las características dominantes de la biomedicina actual, lo que sin duda posibilita la exclusión de las condiciones socio-económicas en la explicación de la causalidad de las enfermedades, en otras palabras se enfatiza en la historia natural de la enfermedad pero sin su historia social, y con total desprecio por el análisis de vulnerabilidad, enfoque de riesgo epidemiológico, participación comunitaria e integralidad. En este sentido se apuntala hacia la realización de planes más que de actividades aisladas, con integración de las distintas aéreas del conocimiento en salud, y con la suma cada vez mayor de voluntades, como pudo apreciarse con el trabajo hasta ahora realizado. 


\section{Bibliografía}

Abramson, J. (1984), “Application of epidemiology in community-oriented primary care”, en: Public Health Reports, volumen 99 número 5, pp. 437442.

Abramson, J. (1988), “Community-oriented primary care: strategy, approaches and practice -a review”, en: Public Health Reviews, volumen 16 número 1-2, pp.35-98.

Andrade, L. (2007), "Responsabilidad Social y Democracia”. La Prensa. Disponible en http://www.google.com. Consultado el 28/05/2011.

Alemany, M., Bernabeu-Mestre, J., Galiana, M., López, C. y TrescastroLópez, E. (2009), Hambre cuando hay pan para todos. Homenaje al profesor José María Bengoa Lecanda (1913-2010). CEE Limencop, S.L. España.

Arouca, S. (1975), “Introdução à crítica do setor saúde”, en: Nêmesis, volumen 1, pp.17-24.

Bosi, M. (2012), "Qualitative research in collective health: overview and challenge”, en: Ciência \& saúde coletiva, volumen 3 número17, pp.575586.

Cashman, S., Anderson, R., Weisbuch, J., Schwarz, M. y Fulmer, H. (1999), "Carrying out the Medicine/Public Health Initiative: the roles of preventive medicine and community-responsive care”, en: Academic Medicine, volumen74, pp. 473-83.

Castel, Robert (1995), La Métamorphoses de la question sociale. Une Chronique du salariat. Fayard, Francia.

De Almeida, N. (2000), La ciencia tímida. Ensayos de deconstrucción de la epidemiología. Editorial Lugar, Buenos Aire.

De Almeida, N., Castiel, L. y Ayres, J. (2009), “Riesgo: concepto básico de la epidemiología”, en: Salud Colectiva, volumen 5 número 3, pp.323-344.

Donabedian, A. (1990), "The seven pillars of quality", en: Archives of pathology \& laboratory medicine, volumen 114, pp.1115-1118.

Ferrara, F., Acebal, E. y Paganini, S. (1972), Medicina de la comunidad. Inter Médica Editorial. Buenos Aires.

Flexner, A. (1910), Medical education in the United States and Canada: a report to the Carnegie Foundation for the advancement of Teaching. The Carnegie Foundation for the Advancement of teaching, New York. 
Frenk, J., Chen, L., Bhutta, Z., Cohen, J., Crisp, N., Evans T., et al. (2010), "Health professionals for a new century: transforming education to strengthen health systems in an interdependent world”, en: The lancet, volumen 376 número 9756, pp.1923-1958.

Gacitúa, E. y Davis, S. (2000), Introducción:“ Pobreza y exclusión social en América Latina y el Caribe”. En, Exclusión social y reducción de la pobreza en América Latina y el Caribe, Editores Gacitúa, E., Sojo, C. Y Davis, S. San José, Costa Rica, FLASCO-Costa Rica/ Banco Mundial.

Geiger, H. (1993), “Community-oriented primary care. The legacy of Sidney Kak”, en: American Journal of Public Health, volume 83 número 7, pp.946-947.

Gofin, J. y Foz, G. (1998), “La orientación comunitaria de la atención primaria: el legado del prof. SL Kark (1911-1998)”, en: Atención Primaria, volumen 23, pp. 136-138.

González, E. (2004), “La misión académica extensión universitaria como promotora de la interacción Universidad- Sociedad”, en: Docencia Universitaria, volumen 5 número 1-2, pp.9-33.

Jiménez-De Gracia, L., Ruiz-Moral, R., Gavilán-Moral, E., Hueso-Montoro, C., Cano-Caballero, D. y Alba-Dios, M. (2012), “Opinions of family doctors on the involvement of patients in the taking of decisions: a study with focus groups”, en: Atención Primaria, volumen 44 número 7, pp.379-384.

Lindman, E. (1951), Community. Encyclopedia of the social sciences. MacMillan Company. Nueva York.

Magill, M. (1999), “Medicine and Public Health. Whose Agenda?” en: Archives of Family Medicine, volumen 8, pp. 81-82.

Marx, C. y Engels, F. (1959), La Ideología Alemana. Editorial Pueblos Unidos, Montevideo.

McWhinney, I. (1981), An Introduction to Comunitary Medicine. Oxford University Press. New York.

Menéndez, E. (1981), Poder, estratificación y salud. Análisis de las condiciones sociales y económicas de la enfermedad en Yucatán. Ediciones de la Casa Chata, México.

Ídem (1984), Descripción y análisis de las representaciones y prácticas de grupos domésticos sobre la mortalidad en niños menores de cinco años en una comunidad de Guanajuato. Ministerio de Salud, México.

Ídem (2003), “Modelos de atención de los padecimientos: de exclusiones teóricas y articulaciones prácticas”, en: Ciencia \& Saude Colectiva, Numero 8 volumen 1, pp. 185-2007. 
Ídem (2005), “Modelo médico y la salud de los trabajadores”, en: Salud Colectiva, la plata. Numero 1 volumen 1, pp, 9-32.

Morin, Edgar. (2003), Introducción al pensamiento Complejo, Editorial Gedisa. Barcelona.

Mullan, F. (1982), “Community-oriented primary care: an agenda for the 80's”, en: The New England Journal of Medicine, volumen 307, pp. 10761078.

Nickens, H. (1999), “It’s about time. The medicine/public health initiative”, en: American journal of preventive medicine, volumen 16, pp.20-21.

Organización Panamericana de la Salud. (2008), La renovación de la atención primaria de salud en las Américas. Numero 2: La formación en medicina orientada hacia la atención primaria en salud. Seminario latinoamericano de educación médica. Belo Horizonte, Brasil, 21 al 24 de julio.

Owens, T., Dominici, L. y Saldaña, A. (1998), ¿Qué es la Medicina Comunitaria?, Universidad de Panamá.

Parsons, T. y Shils, E. (1951), Towards a general theory of action. Harvard University. Press.

Portillo, J. (1994), "Pensamiento social en salud en América Latina Juan César García”, en: Revista Médica del Uruguay, volumen 10, pp. 205-206.

Ruiz-Moral, R., Pérula, L., Muñoz, M., Jiménez, C., González, V., Alba, A., et al. (2011), "Patients' satisfaction with communication with their family doctors: comparison of three methods for assessing unmet needs" en: Revista Española de Salud Pública, volumen 85 número 3, pp. 315-322.

Silva, P. (2009), "Medicina comunitaria. Introducción a un análisis crítico” en: Salud Colectiva, volumen 5 numero 1, pp. 121-126.

Silver, G. (1984), “The practice of community -oriented primary health care”, en: World Health Forum, Volumen 5, pp. 91-91.

Smuts, J. (1926), Holims and Evolution, Nova Lorque. Mac. Millan.

Stewart, I. (2001), ¿Juega Dios a los Dados?, Drakontos. Crítica. Barcelona.

Weil, P. (1997), Holística: una nueva visión y abordaje de lo real, Editorial San Pablo. Bogotá.

Weinstein, L. (1991), “La Racionalidad Integradora. Un aporte a la Salud de la Cultura”, Revista de Psiquiatría, volumen 8, pp. 933-935, 
Polis, Revista Latinoamericana, Volumen 13, $N^{\circ}$ 38, 2014

Welch, W. y Rose, W. (1915), Institute of Hygiene: a report the general education board of Rockefeller Foundation, The Rockefeller Foundation, New York.

Wright, R. (1993), "Community-oriented primary care: the cornerstone of health care reform”, en: JAMA, volumen 269, pp. 2544-2547.

World Health Organization. (2000), World Health Report 2000. World Health Organization, Geneva.

Recibido: 16.05.2013

Aceptado: 10.12.2013 